Wright State University

CORE Scholar

Mathematics and Statistics Faculty

Publications

Mathematics and Statistics

$9-2011$

\title{
The Constructions of Almost Binary Sequence Pairs and Binary Sequence Pairs with Three-Level Autocorrelation
}

Xiuping Peng

Chengqian Xu

Guang Li

Krishnasamy T. Arasu

Wright State University - Main Campus, k.arasu@wright.edu

Follow this and additional works at: https://corescholar.libraries.wright.edu/math

Part of the Applied Mathematics Commons, Applied Statistics Commons, and the Mathematics Commons

\section{Repository Citation}

Peng, X., Xu, C., Li, G., \& Arasu, K. T. (2011). The Constructions of Almost Binary Sequence Pairs and Binary Sequence Pairs with Three-Level Autocorrelation. IEICE Transactions on Fundamentals of Electronics Communications and Computer Sciences, E94-A (9), 1886-1891.

https://corescholar.libraries.wright.edu/math/63

This Article is brought to you for free and open access by the Mathematics and Statistics department at CORE Scholar. It has been accepted for inclusion in Mathematics and Statistics Faculty Publications by an authorized administrator of CORE Scholar. For more information, please contact library-corescholar@wright.edu. 


\title{
LETTER \\ The Constructions of Almost Binary Sequence Pairs and Binary Sequence Pairs with Three-Level Autocorrelation*
}

\author{
Xiuping PENG ${ }^{\dagger a)}$, Chengqian XU ${ }^{\dagger}$, Gang LI ${ }^{\dagger}$, Nonmembers, Kai LIU ${ }^{\dagger}$, Member, \\ and Krishnasamy Thiru ARASU ${ }^{\text {t† }}$, Nonmember
}

SUMMARY In this letter, a new class of almost binary sequence pairs with a single zero element and three autocorrelation values is presented. The new almost binary sequence pairs are based on cyclic difference sets and difference set pairs. By applying the method to the binary sequence pairs, new binary sequence pairs with three-level autocorrelation are constructed. It is shown that new sequence pairs from our constructions are balanced or almost balanced and have optimal three-level autocorrelation when the characteristic sequences or sequence pairs of difference sets or difference-set pairs are balanced or almost balanced and have optimal autocorrelations.

key words: autocorrelation, difference sets, difference set pairs, almost binary sequence pairs, binary sequence pairs

\section{Introduction}

Pseudorandom binary sequences $\{x(t)\}$, where $x(t) \in$ $\{+1,-1\}$, of period $N$ are widely used in code-division multiple-access (CDMA) mobile communications and stream-cipher systems due to their randomness and simplicity in their generation. For these sequences the transmitted and received signals are the same to extract the useful signals using the periodic autocorrelation, and it is desirable to use binary sequences with nontrivial autocorrelation as low as possible. Additionally, depending on the applications, mismatched filtering sequences were proposed [1]. In mismatched filtering systems, the receiver uses spread frequency sequences different from the sequences in the transmitter as the filtering sequence to compress pulses. Comparing matched filtering with the same address code sequences in the transmitter and receiver, we found out that mismatched filtering can obtain more spread frequency sequences in the precondition of ensuring acceptable SNR loss. A sequence pair which is a class of discrete signal can be considered as a class of mismatched sequences [2]. If the PACF of the sequence pair side-lobes are all zero, we say the sequence pair is a perfect sequence pair. Since the known existence result for perfect binary sequence pairs is

\footnotetext{
Manuscript received December 7, 2010.

Manuscript revised May 16, 2011.

${ }^{\dagger}$ The authors are with the College of Information Science and Engineering, Yanshan University, Qinhuangdao 066004 China.

† The author is with the Department of Mathematics and Statistics, Wright State University, Dayton, Ohio45435 USA.

*This paper was supported by China Nation Natural Science Foundation $(60872061,60971126)$, by Natural Science Foundation of Hebei Province, China (No. F2008000855) and by Department of Education Foundation of Hebei Province, China (2010286).

a) E-mail: honphin@ysu.edu.cn

DOI: $10.1587 /$ transfun.E94.A.1886
}

only for even period, Jiang extended the study to punctured binary sequence pairs in [3] and $\mathrm{Xu}$ to the ternary and quaternary sequence pairs in [4] and [5].

Recently, the concepts of almost binary and almost quadriphase sequences (sequences with a signal zero element) were proposed alternatively, and several interesting constructions have been reported [6], [7]. In this letter, by applying the method to the binary sequence pairs; we present a class of almost binary sequence pairs with threelevel autocorrelation, which is based on difference sets and difference set pairs and has optimal balance among +1 's and -1 's. Additionally, we discuss the binary sequence pairs by replacing $x(\alpha)=1$ with $x(\alpha)=-1$ or $x(\alpha)=-1$ with $x(\alpha)=1$ for $0 \leq \alpha \leq N-1$.

The outline of the letter is as follows. Section 2 gives some preliminaries for our presentation. In Sect. 3, we present the methods to construct almost binary sequence pairs based on difference sets and difference set pairs, which preserve the autocorrelation and balance property of the given binary sequence or sequence pair. Applying the method to the binary sequence pairs, new binary sequence pairs with three-level autocorrelation are constructed in Sect. 4. Finally, some concluding remarks are given in Sect. 5 .

\section{Preliminaries}

We review some notations in this section.

Let $D$ be a subset of $Z_{N}$. The characteristic sequence $s(t)$ of $D$ is defined as

$$
s(t)= \begin{cases}-1, & \text { if } t \bmod N \in D \\ 1, & \text { otherwise. }\end{cases}
$$

Let $U$ and $V$ be a subset of $Z_{N}$, and let $U$ and $V$ be the characteristic sets of the binary sequences $x^{\prime}$ and $y^{\prime}$ respectively, then define an almost binary sequence pair $(x, y)$ as follows:

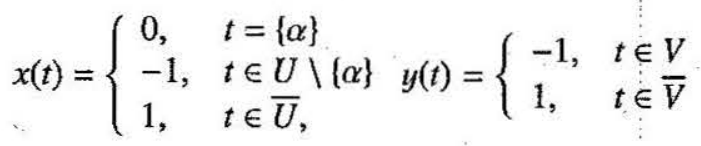

where $\alpha \in Z_{N}$.

Let $(x, y)$ be an almost binary sequence pair of period $N$. Then the periodic autocorrelation function (PACF) of the almost binary sequence pair $(x, y)$ with three-level autocorrelation at shift $0 \leq \tau<N$ is defined as 


$$
\begin{aligned}
R_{(x, y)}(\tau) & =\sum_{j=0}^{N-1} x(j) y(j+\tau) \\
& = \begin{cases}F, & \tau=0 \text { and } F \neq E_{1} \neq E_{2} \\
E_{1}, & \tau \in H \\
E_{2}, & \tau \in Z_{N}^{*}-H\end{cases}
\end{aligned}
$$

where $j+\tau=(j+\tau) \bmod N, H$ is a subset of $Z_{N}^{*}$.

Let $\left\{x^{\prime}(t)\right\}$ and $\left\{y^{\prime}(t)\right\}$ be two binary sequences of period $N$, and let the sets $U$ and $V$ be the characteristic sets of the sequences $\left\{x^{\prime}(t)\right\}$ and $\left\{y^{\prime}(t)\right\}$. The autocorrelation property of $\left\{x^{\prime}(t)\right\}$ and $\left\{y^{\prime}(t)\right\}$ are determined by the difference function and defined as

$$
d_{U, V}(\tau)=|(\tau+U) \cap V|
$$

Let $D$ be a subset of $Z_{N}$, and let $k=|D| . D$ is called a $(N, k, \lambda)$ difference set if the equation $d-d^{\prime}=\omega$ has $\lambda$ solutions $\left(d, d^{\prime}\right) \in D \times D$ for each nonzero element of $Z_{N}$.

Let $U=\left\{u_{i} \mid 1 \leq i \leq k\right\} \subset Z_{N}$ and $V=\left\{v_{i} \mid 1 \leq i \leq k^{\prime}\right\} \subset$ $Z_{N}$ be two subsets of $Z_{N}$ with $k$ and $k^{\prime}$ elements respectively. Let $e=|U \cap V|$. Then $(U, V)$ is called an $\left(N, k, k^{\prime}, e, \lambda\right)$ difference set pair if every nonzero $g \in Z_{N}^{*}, g$ can be expressed in exactly $\lambda$ ways in the form $u_{i}-v_{j} \equiv g$, where $u_{i} \in U$ and $v_{j} \in V$. Obviously, if $U=V$, the difference set pair is very natural generalization of cyclic difference set.

Lemma 1([8]): Let $(U, V)$ be a $\left(N, k, k^{\prime}, e, \lambda\right)$ difference set pair of $Z_{N}$. Then

1) $(U, \bar{V})$ is a $\left(N, k, N-k^{\prime}, k-e, k-\lambda\right)$ difference set pair and $(\bar{U}, V)$ is a $\left(N, N-k, k^{\prime}, k^{\prime}-e, k^{\prime}-\lambda\right)$ difference set pair, where $\bar{U}$ and $\bar{V}$ are defined as $\bar{U}=Z_{N}-U$ and $\bar{V}=Z_{N}-V$, and are called the complement of $U$ and $V$.

2) $(\bar{U}, \bar{V})$ is a $\left(N, N-k, N-k^{\prime}, e^{\prime}, \lambda^{\prime}\right)$ difference set pair, where $e^{\prime}=N-\left(k+k^{\prime}\right)+e$ and $\lambda^{\prime}=N-\left(k+k^{\prime}\right)+\lambda$.

As mentioned in paper [9], the binary sequence pair $\left(x^{\prime}, y^{\prime}\right)$ has a two-level autocorrelation function if and only if its characteristic set pair $(U, V)$ is a difference set pair. Thus finding binary sequence pairs with two-level autocorrelation function of period $N$ is the same as searching for difference set pairs of $Z_{N}$. Then the relationship between the parameters $\left(N, k, k^{\prime}, e, \lambda\right)$ of difference set pair $(U, V)$ and PACF of binary sequence pair $\left(x^{\prime}, y^{\prime}\right)$ is as follows.

Lemma 2([9]): Let $U$ and $V$ be two subsets of $Z_{N}$, and $x^{\prime}=$ $\left(x^{\prime}(0), x^{\prime}(1), \cdots, x^{\prime}(N-1)\right)$ and $y^{\prime}=\left(y^{\prime}(0), y^{\prime}(1), \cdots, y^{\prime}(N-\right.$ 1)) be two characteristic sequences of $U$ and $V$ respectively. Then the relationship between the parameters $\left(N, k, k^{\prime}, e, \lambda\right)$ of difference set pair $(U, V)$ and PACF of binary sequence pair $\left(x^{\prime}, y^{\prime}\right)$ is

$$
R_{\left(x^{\prime}, y^{\prime}\right)}(\tau)= \begin{cases}N-2\left(k+k^{\prime}\right)+4 e, & \text { for } \tau=0 \\ N-2\left(k+k^{\prime}\right)+4 \lambda, & \text { for } \tau \neq 0 .\end{cases}
$$

\section{Constructions of Almost Binary Sequence Pairs}

In this subsection, two constructions of almost binary sequence pairs are presented.

\subsection{Construction Based on Difference Sets}

Theorem 1: Let the set $D$ be a cyclic difference set with parameters $(N, k, \lambda)$, and let

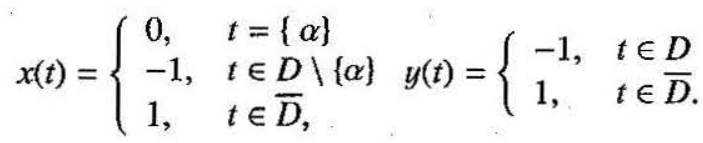

Then the sequence pair $(x, y)$ is an almost binary sequence pair with three-level autocorrelation.

Proof: By the definition, the autocorrelation function of the sequence pair becomes

$$
\begin{aligned}
R_{(x, y)}(\tau)= & |\{t: t \in D \backslash\{\alpha\}, t+\tau \in D\}| \\
& +|\{t: t \in \bar{D}, t+\tau \in \bar{D}\}| \\
& -|\{t: t \in D \backslash\{\alpha\}, t+\tau \in \bar{D}\}| \\
& -|\{t: t \in \bar{D}, t+\tau \in D\}| \\
= & |\{t: t \in D, t+\tau \in D\}|+|\{t: t \in \bar{D}, t+\tau \in \bar{D}\}| \\
& -|\{t: t \in D, t+\tau \in \bar{D}\}|-|\{t: t \in \bar{D}, t+\tau \in D\}| \\
& -|\{t: t \in\{\alpha\}, t+\tau \in D\}|+|\{t: t \in\{\alpha\}, t+\tau \in \bar{D}\}| \\
= & d_{D}(\tau)+d_{\bar{D}}(\tau)-d_{D, \bar{D}}(\tau)-d_{\bar{D}, D}(\tau) \\
& -|\{t: t \in\{\alpha\}, t+\tau \in D\}|+|\{t: t \in\{\alpha\}, t+\tau \in \bar{D}\}|
\end{aligned}
$$

For $\tau=0, R_{(x, y)}=N-1$ is the trivial value.

For $\tau \neq 0$, firstly, we come to consider

$$
\Delta=d_{D}(\tau)+d_{\bar{D}}(\tau)-d_{D, \bar{D}}(\tau)-d_{\bar{D}, D}(\tau)
$$

From Lemma 1, we can get $\Delta=N-4(k-\lambda)$. Furthermore, when $\tau \in D-\alpha$, it is easily checked that $\{t: t \in\{\alpha\}, t+\tau \in$ $\bar{D}] \mid=0$ and $|\{t: t \in\{\alpha\}, t+\tau \in D\}|=1$, and we obtain $|\{t: t \in\{\alpha\}, t+\tau \in \bar{D}\}|=1$ and $|\{t: t \in\{\alpha\}, t+\tau \in D\}|=0$ for the case $\tau \in \bar{D}-\alpha$.

Concluding from the above, the almost binary sequence pair $(x, y)$ has the following three autocorrelation values:

$$
R_{(x, y)}(\tau)= \begin{cases}N-1, & \text { for } t=\{\alpha\} \\ N-4(k-\lambda)-1, & \text { for } t \in D-\alpha \\ N-4(k-\lambda)+1, & \text { for } t \in \bar{D}-\alpha\end{cases}
$$

It is obvious that we can use all kinds of difference sets, such as Singer set, Hadamard set, Twin set, Cyclotomic set and so on, to construct the almost binary sequence pairs.

Using Singer set, an example of the above theorem is given as follows.

Example 1: Let $D$ be a Singer set of $Z_{63}$ with parameters $(63,31,15)$, and

$D=\{0,1,2,3,4,8,9,10,11,13,14,16,17,18,21,24,25$,
$27,30,32,36,41,42,45,46,47,49,51,53,54,61\}$.

By Theorem 1, if we let $x(4)=0$. The autocorrelation function of the almost binary sequence pair

$$
\begin{aligned}
(x, y)= & (----0+++----+--+---++ \\
& -++--+-++-+-+++-++++- \\
& -++---+-+-+--++++++-+,
\end{aligned}
$$




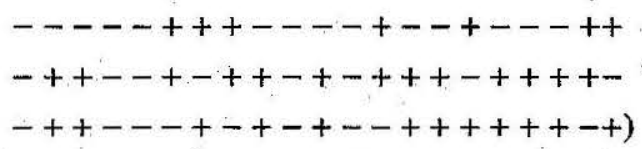

is as follows:

$$
\begin{aligned}
R_{(x, y)}(\tau)= & {[62,0,0,0,-2,-2,-2,-2,0,-2,-2,0,-2,-2,} \\
& -2,0,0,-2,0,0,-2,-2,0,-2,0,0,-2,0,-2,0, \\
& 0,0,-2,0,0,0,0,-2,-2,0,0,-2,-2,-2,0,-2,0, \\
& -2,0,-2,-2,0,0,0,0,0,0,-2,0,-2,-2,-2,-2\}
\end{aligned}
$$

It is easily checked that the numbers of occurrence of +1 in $x$ is $32,-1$ is 30 and +1 in $y$ is $32,-1$ is 31 .

In the following, we consider another construction of almost binary sequence pairs which is based on difference set pairs:

\subsection{Constructions Based on Difference Set Pairs}

Theorem 2: Let the set $(U, V)$ be a cyclic difference set pair with parameters $\left(N, k, k^{\prime}, e, \lambda\right)$, and let

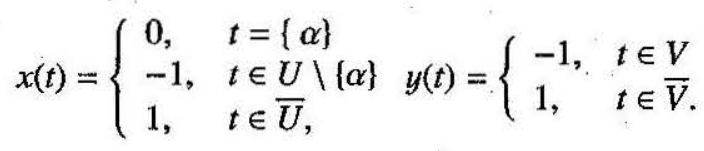

where $\alpha \notin U \cap V$. Then the sequence pair $(x, y)$ is an almost binary sequence pair with three-level autocorrelation.

Proof: As before, we need only to consider the autocorrelation function of the sequence pair.

$$
\begin{aligned}
R_{(x, y)}(\tau)= & |\{t: t \in U, t+\tau \in V\}|+|\{t: t \in \bar{U}, t+\tau \in \bar{V}\}| \\
& -|\{t: t \in U, t+\tau \in \bar{V}\}|-|\{t: t \in \bar{U}, t+\tau \in V\}| \\
& -|\{t: t \in\{\alpha\}, t+\tau \in V\}|+|\{t: t \in\{\alpha\}, t+\tau \in \bar{V}\}| .
\end{aligned}
$$

For $\tau=0$, since $\alpha \notin U \cap V$, then the elements $x(\alpha)$ and $y(\alpha)$ of the characteristic sequence pair of $(U, V)$ satisfy $x(\alpha)$. $y(\alpha)=-1$. By Theorem 2, we can obtain $x(\alpha) \cdot y(\alpha)=0$. From Lemma 2, we have $R_{(x, y)}(0)=N-2\left(k+k^{\prime}\right)+4 e+1$. For $\tau \neq 0$, we come to consider

$$
\Delta=d_{U, V}(\tau)+d_{\bar{U}, \bar{V}}(\tau)-d_{U, \bar{V}}(\tau)-d_{(\bar{U}, V)}(\tau) .
$$

From Lemma 1 , we can get $\Delta=N-2\left(k+k^{\prime}\right)+4 \lambda$. Furthermore, when $\tau \in V-\alpha$, it is easily checked that $\{t: t \in$ $\{\alpha\}, t+\tau \in \bar{V}\} \mid=0$ and $|\{t: t \in\{\alpha\}, t+\tau \in V\}|=1$, and we obtain $|\{t: t \in\{0\}, t+\tau \in \bar{V}\}|=1$ and $|\{t: t \in\{0\}, t+\tau \in V\}|=0$ for the case $\tau \in \vec{V}-\alpha$.

Concluding from the above, the almost binary sequence pair $(x, y)$ has the following three autocorrelation values:

$$
R_{(x, y)}(\tau)=\left\{\begin{array}{l}
N-2\left(k+k^{\prime}\right)+4 e+1, \text { for } \tau=0 \\
N-2\left(k+k^{\prime}\right)+4 \lambda-1, \text { for } \tau \in V-\alpha \\
N-2\left(k+k^{\prime}\right)+4 \lambda+1, \text { for } \tau \in \bar{V}-\alpha .
\end{array}\right.
$$

For the case when $\alpha \in U \cap V$, we can also get the following result.
Corollary 1: Let the set $(U, V)$ be a cyclic difference set pair with parameters $\left(N, k, k^{\prime}, e, \lambda\right)$, and let

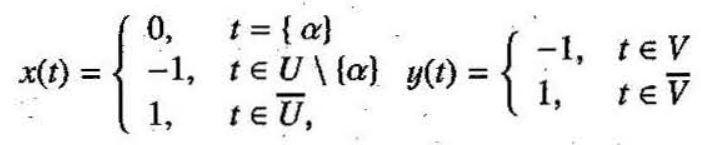

where $\alpha \in U \cap V$. Then the sequence pair $(x, y)$ is an almost binary sequence pair with three-level autocorrelation.

Proof: When $\tau=0$, since $\alpha \in U \cap V$, then the elements $x(\alpha)$ and $y(\alpha)$ of the characteristic sequence pair of $(U, V)$ satisfy $x(\alpha) \cdot y(\alpha)=1$. By. Theorem 3, we can obtain $x(\alpha) \cdot y(\alpha)=0$. From Lemma 2, we have $R_{(x, y)}(0)=N-2\left(k+k^{\prime}\right)+4 e-1$. Then the new sequence pair induced from the construction has three-level autocorrelation as shown in the following.

$$
R_{(x, y)}(\tau)=\left\{\begin{array}{l}
N-2\left(k+k^{\prime}\right)+4 e-1, \text { for } \tau=0 \\
N-2\left(k+k^{\prime}\right)+4 \lambda-1, \text { for } \tau \in V-\alpha \\
N-2\left(k+k^{\prime}\right)+4 \lambda+1, \text { for } \tau \in \bar{V}-\alpha .
\end{array}\right.
$$

Example 2: Let a difference set pair $(U, V)$ with parameters $(21,11,8,8,4)$ be given by

$$
\begin{aligned}
& U=\{5,6,8,11,12,15,18,20\} \\
& V=\{5,6,7,8,10,11,12,15,16,18,20\} .
\end{aligned}
$$

By Theorem 2, if we let $y(7)=0$, the almost binary sequence pair $(x, y)$ of period 21 is given by

$$
\begin{aligned}
(x, y)= & (+++++--+-++--++-++-+-, \\
& +++++--0-+---++--+-+-) .
\end{aligned}
$$

The autocorrelations of the sequence pair are given by

$$
\begin{aligned}
R_{(x, y)}= & \{16,-2,-2,0,0,0,0,0,-2,0,-2,0,0, \\
& -2,0,0,-2,-2,0,0,-2\} .
\end{aligned}
$$

By Corollary 1, if we let $x(5)=0$, the autocorrelations of the almost binary sequence pair $(x, y)$ of period 21 are given by

$$
\begin{aligned}
R_{(x, y)}= & \{14,-2,-2,-2,0,-2,-2,-2,0,0,-2,-2, \\
& 0,-2,0,-2,0,0,0,0,0\} .
\end{aligned}
$$

\section{Constructions of Binary Sequence Pairs}

In the previous section, we discuss the resultant almost binary sequence pairs by replacing $x(\alpha)=1$ or -1 with $x(\alpha)=0$. In this section, we will discuss the binary sequence pairs with three-level autocorrelation by replacing $x(\alpha)=-1$ with $x(\alpha)=1$, and vice versa.

\subsection{Construction Based on Difference Sets}

Theorem 3: Let the set $D$ be a cyclic difference set with parameters $(N, k, \lambda)$, and let

$$
x(t)=\left\{\begin{array}{ll}
1, & t=\{\alpha\} \\
-1, & t \in D \backslash\{\alpha\} \\
1, & t \in \bar{D},
\end{array} \quad y(t)= \begin{cases}-1, & t \in D \\
1, & t \in \bar{D} .\end{cases}\right.
$$


Then the sequence pair $(x, y)$ is a binary sequence pair with three-level autocorrelation.

Proof: Similar to Theorem1, the autocorrelation function of the binary sequence pair becomes

$$
\begin{aligned}
R_{(x, y)}(\tau)= & d_{D}(\tau)+d_{\bar{D}}(\tau)-d_{D, \bar{D}}(\tau)-d_{\bar{D}, D}(\tau) \\
& -2|\{t: t \in\{\alpha\}, t+\tau \in D\}| \\
& +2|\{t: t \in\{\alpha\}, t+\tau \in \bar{D}\}| .
\end{aligned}
$$

For $\tau=0$, since $x(\alpha)=1$, then $R_{(x, y)}(0)=N-2$.

For $\tau \neq 0$, we come to consider

$$
\Delta=d_{D}(\tau)+d_{\bar{D}}(\tau)-d_{D, \bar{D}}(\tau)-d_{\bar{D}, D}(\tau)=N-4(k-\lambda) .
$$

Furthermore, when $\tau \in D-\alpha$, it is easily checked that $\mid\{t$ : $t \in\{\alpha\}, t+\tau \in \bar{D}\} \mid=0$ and $|\{t: t \in\{\alpha\}, t+\tau \in D\}|=1$, and we obtain $\mid\{t: t \in\{\alpha\}, t+\tau \in \bar{D}\}\}=1$ and $\mid\{t: t \in\{\alpha\}, t+\tau \in$ $D\} \mid=0$ for the case $\tau \in \bar{D}-\alpha$.

Concluding from the above, the binary sequence pair $(x, y)$ has the following three autocorrelation values:

$$
R_{(x, y)}(\tau)= \begin{cases}N-2, & \text { for } \tau=0 \\ N-4(k-\lambda)-2, & \text { for } \tau \in D-\alpha \\ N-4(k-\lambda)+2, & \text { for } \tau \in \bar{D}-\alpha .\end{cases}
$$

Clearly, when the element of the characteristic sequence of the difference set +1 is replaced with -1 , we can also construct a binary sequence pair with three-level autocorrelation as the following.

Corollary 2: Let the set $D$ be a cyclic difference set with parameters $(N, k, \lambda)$, and let

$$
x(t)=\left\{\begin{array}{ll}
-1, & t=\{\alpha\} \\
-1, & t \in D \\
1, & t \in \bar{D} \backslash\{\alpha\},
\end{array} \quad y(t)= \begin{cases}-1, & t \in D \\
1, & t \in \bar{D} .\end{cases}\right.
$$

Then the sequence pair $(x, y)$ is a binary sequence pair with three-level autocorrelation.

The sequence pair generated from Corollary 2 has almost the same properties as the sequence pair from Theorem 3. In the following, we state its correlation property without proof.

$$
R_{(x, y)}(\tau)= \begin{cases}N-2, & \text { for } \tau=0 \\ N-4(k-\lambda)+2, & \text { for } \tau \in D-\alpha \\ N-4(k-\lambda)-2, & \text { for } \tau \in \bar{D}-\alpha .\end{cases}
$$

Example 3: Let $D$ be a Singer set of $Z_{63}$ with parameters $(63,31,15)$, and

$D=\{0,1,2,3,4,8,9,10,11,13,14,16,17,18,21,24,25$,

$27,30,32,36,41,42,45,46,47,49,51,53,54,61\}$.

By Theorem 3, let $x(4)=1$. The autocorrelation function of binary sequence pair

$$
\begin{aligned}
(x, y)= & (----++++----+--+---++ \\
& -++--+-++-+-+++-++++- \\
& -++--+-+-+--++++++-+
\end{aligned}
$$

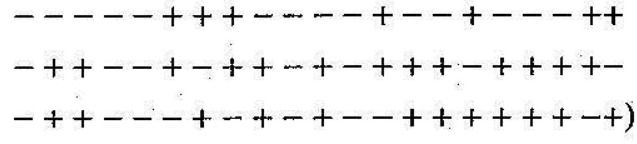

is as follows:

$$
\begin{aligned}
R_{(x, y)}(\tau) & \\
= & \{61,1,1,1,1,-3,-3,-3,-3,1,-3,-3,1,-3,-3, \\
& -3,1,1,-3,1,1,-3,-3,1,-3,1,1,-3,1,-3,1,1,1, \\
& -3,1,1,1,1,-3,-3,1,1,-3,-3,-3,1,-3,1,-3,1, \\
& -3,-3,1,1,1,1,1,1,-3,1,-3,-3,-3,-3\} .
\end{aligned}
$$

By Corollary 2, let $x(5)=-1$. The autocorrelation function of binary sequence pair

$$
\begin{aligned}
(x, y)= & (-----++----+--+---++ \\
& -++--+-++-+-+++-++++- \\
& -++---+-+-+--++++++-+, \\
& -----+++----+--+---++ \\
& -++--+-++-+-+++-++++- \\
& -++---+-+-+--++++++-+)
\end{aligned}
$$

is as follows:

$$
\begin{aligned}
R_{(x, y)}(\tau) & \\
= & \{61,-3,-3,1,1,1,1,-3,1,1,-3,1,1,1,-3,-3,1,-3, \\
& -3,1,1,-3,1,-3,-3,1,-3,1,-3,-3,-3,1,-3,-3, \\
& -3,-3,1,1,-3,-3,1,1,1,-3,1,-3,1,-3,1,1,-3, \\
& -3,-3,-3,-3,-3,1,-3,1,1,1,1,1\} .
\end{aligned}
$$

In the following, applying the construction based on difference set to difference set pair, we can also construct a binary sequence pair with three-level autocorrelation.

\subsection{Construction Based on Difference Set Pairs -}

Theorem 4: Let the set $(U, V)$ be a cyclic difference set pair with parameters $\left(N, k, k^{\prime}, e, \lambda\right)$, and let

$$
x(t)=\left\{\begin{array}{ll}
1, & t=\{\alpha\} \\
-1, & t \in U \backslash\{\alpha\} \\
1, & t \in \bar{U},
\end{array} y(t)= \begin{cases}-1, & t \in V \\
1, & t \in \bar{V}\end{cases}\right.
$$

where $\alpha \notin U \cap V$. Then the sequence pair $(x, y)$ is a binary sequence pair with three-level autocorrelation.

Proof: Similar to Theorem 2, the autocorrelation function of the binary sequence pair becomes

$$
\begin{aligned}
R_{(x, y)}(\tau)= & d_{U, V}(\tau)+d_{\bar{U}, \bar{V}}(\tau)-d_{U, \bar{V}}(\tau)-d_{\overline{(U, V)}}(\tau) \\
& -2|\{t: t \in\{\alpha\}, t+\tau \in V\}| \\
& +2|\{t: t \in\{\alpha\}, t+\tau \in \bar{V}\}| .
\end{aligned}
$$

For $\tau=0$, since $\alpha \notin U \cap V$, then the elements $x(\alpha)$ and $y(\alpha)$ of the characteristic sequence pair of $(U, V)$ satisfy $x(\alpha) \cdot y(\alpha)=-1$. By Theorem 4, we can obtain $x(\alpha) \cdot y(\alpha) \stackrel{=}{=}$ 
1. Then we have, $R_{(x, y)}(0)=N-2\left(k+k^{\prime}\right)+4 e+2$.

For $\tau \neq 0$, and when $\tau \in V-\alpha$, it is easily got that $R_{(x, y)}=N-2\left(k+k^{\prime}\right)+4 \lambda-2$, and we obtain $R_{(x, y)}=N-$ $2\left(k+k^{\prime}\right)+4 \lambda+2$ for the case $\tau \in \bar{V}-\alpha$.

Concluding from the above, the binary sequence pair $(x, y)$ has the following three autocorrelation values:

$$
R_{(x, y)}(\tau)=\left\{\begin{array}{l}
N-2\left(k+k^{\prime}\right)+4 e+2, \text { for } \tau=0 \\
N-2\left(k+k^{\prime}\right)+4 \lambda-2, \text { for } \tau \in V-\alpha \\
N-2\left(k+k^{\prime}\right)+4 \lambda+2, \text { for } \tau \in \bar{V}-\alpha
\end{array}\right.
$$

For the case $\alpha \in U \cap V$, we can also get the following result.

Corollary 3: Let the set $(U, V)$ be a cyclic difference set pair with parameters $\left(N, k, k^{\prime}, e, \lambda\right)$, and let

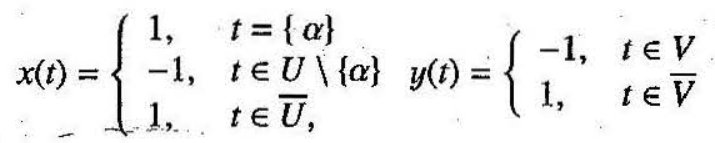

where $\alpha \in U \cap V$. Then the sequence pair $(x, y)$ is a binary sequence pair with three-level autocorrelation.

Proof: For $\tau=0$, since $\alpha \in U \cap V$, we can get $R_{(x, y)}(0)=$ $N-2\left(k+k^{\prime}\right)+4 e-2$. Then the new sequence pair induced from the construction have three-level autocorrelation as the following.

$$
R_{(x, y)}(\tau)=\left\{\begin{array}{l}
N-2\left(k+k^{\prime}\right)+4 e-2, \text { for } \tau=0 \\
N-2\left(k+k^{\prime}\right)+4 \lambda-2, \text { for } \tau \in V-\alpha \\
N-2\left(k+k^{\prime}\right)+4 \lambda+2, \text { for } \tau \in \bar{V}-\alpha .
\end{array}\right.
$$

Note that the binary sequence pairs constructed in Theorem 4 and Corollary 3 by replacing the element of the characteristic sequence pair of difference set pair -1 with +1 , we can also get another kind of binary sequence pair with three-level autocorrelation by replacing +1 with -1 in the following.

Corollary 4: Let the set $(U, V)$ be a cyclic difference set pair with parameters $\left(N, k, k^{\prime}, e, \lambda\right)$, and let

$$
x(t)=\left\{\begin{array}{ll}
-1, & t \in U \cup\{\alpha\} \\
1, & t \in \bar{U} \backslash\{\alpha\},
\end{array} \quad y(t)= \begin{cases}-1, & t \in V \\
1, & t \in \bar{V}\end{cases}\right.
$$

where $\alpha \in \bar{U} \cap V$. Then the sequence pair $(x, y)$ is a binary sequence pair with three-level autocorrelation.

Proof: As before, the autocorrelation function of the binary sequence pair becomes

$$
\begin{aligned}
R_{(x, y)}(\tau)= & d_{U, V}(\tau)+d_{\bar{U}, \bar{V}}(\tau)-d_{V, \bar{V}}(\tau)-d_{(\bar{U}, V)}(\tau) \\
& +2|\{t: t \in\{\alpha\}, t+\tau \in V\}| \\
& -2|\{t: t \in\{\alpha\}, t+\tau \in \bar{V}\}| .
\end{aligned}
$$

Then the new sequence pair induced from the construction has three-level autocorrelation as the following.

$$
R_{(x, y)}(\tau)=\left\{\begin{array}{l}
N-2\left(k+k^{\prime}\right)+4 e+2, \text { for } \tau=0 \\
N-2\left(k+k^{\prime}\right)+4 \lambda-2, \text { for } \tau \in \bar{V}-\alpha \\
N-2\left(k+k^{\prime}\right)+4 \lambda+2, \text { for } \tau \in V-\alpha .
\end{array}\right.
$$

Corollary 5: Let the set $(U, V)$ be a cyclic difference set pair with parameters $\left(N, k, k^{\prime}, e, \lambda\right)$, and let

$$
x(t)=\left\{\begin{array}{ll}
-1, & t \in U \cup\{\alpha\} \\
1, & t \in \bar{U} \backslash\{\alpha\},
\end{array} y(t)= \begin{cases}-1, & t \in V \\
1, & t \in \bar{V}\end{cases}\right.
$$

where $\alpha \notin \bar{U} \cap V$. Then the sequence pair $(x, y)$ is a binary sequence pair with three-level autocorrelation.

Proof: Similar to Corollary 3, we can get the new sequence pair induced from the construction which has three-level autocorrelation as the following.

$$
R_{(x, y)}(\tau)=\left\{\begin{array}{l}
N-2\left(k+k^{\prime}\right)+4 e-2, \text { for } \tau=0 \\
N-2\left(k+k^{\prime}\right)+4 \lambda-2, \text { for } \tau \in \bar{V}-\alpha \\
N-2\left(k+k^{\prime}\right)+4 \lambda+2, \text { for } \tau \in V-\alpha .
\end{array}\right.
$$

Example 4: Let a difference set pair $(U, V)$ with parameters $(21,11,8,8,4)$ be given by

$$
\begin{aligned}
& U=\{5,6,8,11,12,15,18,20\} \\
& V=\{5,6,7,8,10,11,12,15,16,18,20\} .
\end{aligned}
$$

By Theorem 4 , if we let $y(7)=1$, the binary sequence pair $(x, y)$ of period 21 is given by

$$
\begin{aligned}
(x, y)= & (+++++--+-++--++-++-+- \\
& +++++--+-+---++--+-+-) .
\end{aligned}
$$

The autocorrelations of the sequence pair are given by

$$
\begin{aligned}
R_{(x, y)}= & \{17,-3,-3,1,1,1,1,1,-3,1,-3,1,1, \\
& -3,1,1,-3,-3,1,1,-3\} .
\end{aligned}
$$

By Corollary 3 , if we let $x(5)=1$, the binary sequence pair $(x, y)$ of period 21 is given by

$$
\begin{aligned}
(x, y)= & (++++++-+-++--++-++-+- \\
& +++++----+---++--+-+-) .
\end{aligned}
$$

The autocorrelations of the sequence pair are given by

$$
\begin{aligned}
R_{(x, y)}= & \{13,-3,-3,-3,1,-3,-3,-3,1,1,-3,-3, \\
& 1,-3,1,-3,1,1,1,1,1\} .
\end{aligned}
$$

By Corollary 4 , if we let $x(7)=-1$, the binary sequence pair $(x, y)$ of period 21 is given by

$$
\begin{aligned}
(x, y)= & (+++++---++--++-++-+- \\
& +++++----+---++--+-+-) .
\end{aligned}
$$

The autocorrelations of the sequence pair are given by

$$
\begin{aligned}
R_{(x, y)}= & \{17,1,-3,1,1,1,-3,-3,1,1,-3,1,-3, \\
& 1,-3,-3,-3,-3,-3,1,1\} .
\end{aligned}
$$

By Corollary 5 , if we let $x(4)=-1$, the binary sequence pair $(x, y)$ of period 21 is given by

$$
\begin{aligned}
(x, y)= & (++++---+-++--++-++-+- \\
& +++++----+---++--+-+-) .
\end{aligned}
$$


The autocorrelations of the sequence pair are given by

$$
\begin{gathered}
R_{(x, y)}=\{13,1,1,1,1,-3,1,1,1,-3,-3,1 ; \\
1,-3,1,-3,1,-3,-3,-3,-3\} .
\end{gathered}
$$

\section{Conclusion}

In this letter, some new classes of almost binary sequence pair and binary sequence pair with three-level autocorrelation are proposed. Those almost binary sequence pairs and binary sequence pairs are constructed based on difference sets or difference set pairs with optimum autocorrelation and optimum balance between +1 's and -1 's, when the used characteristic sequences or sequence pairs have optimum autocorrelation and optimum balance of +1 's and -1 's.

It is suggested that the even period binary sequence pair has even out-of-phase correlation values and the odd period binary sequence pair has odd out-of-phase correlation values in Theorem 1 of paper [2]. While in this paper, we can get the binary sequence pairs with even out-of-phase correlation function such as $\{0,-2\}$ of odd period by adding one zero element to the binary sequence pair. So we can get more signals which can be used in the engineering.

As mentioned in paper [10], finding binary sequence pairs of period $N$ with three-level autocorrelation is equivalent to finding almost difference set pairs of $Z_{N}$ with corresponding parameters. Then, it is easy to know that these classes of binary sequence pairs with three-level autocorrelation as pointed out in Sect. 4 are equivalent to some classes of almost difference set pairs. And we can know that difference sets and difference set pairs can be used to construct almost difference set pairs by removing one element or adding one element from this paper. While in paper [10], we can get another kind of almost difference set pairs based on the cyclotomic classes of order 2,4 , and 6 .

We are pleased to note that many families of difference sets have been constructed so far. However, so far only a small number of classes of difference set pairs are found. Numerical results show that there should be more families of difference set pairs remaining to be discovered. For example, the set

$$
(\{0,3,5,8\},\{0,1,3,5,7,8,9,14\})
$$

is a $(15,8,4,4,2)$ difference set pair of $Z_{15}$ which does not belong to any known class of difference set pairs. Another example is the following difference set pair of $Z_{51}$ with parameters $(51,26,16,16,8)$ :

$(\{9,11,14,15,18,24,26,27,32,33,35,41,44,45,48,50\}$, $\{9,11,13,14,18,19,21,22,24,25,26,27,32,33,34,35$, $37,38,40,41,44,45,46,48,50\})$.

It is an open question whether $(3(4 m+1), 6 m+2,4 m, 4 m, 2 m)$ difference set pairs exist for all $m$ which makes $4 m+1$ a prime. It is very likely that the answer is positive.

\section{Acknowledgments}

The authors wish to thank the anonymous reviewers for their detailed and constructive comments which are very helpful to the improvement of this paper.

\section{References}

[1] H. Rohling and W. Plagge, "Mismatched filter design for periodical binary phased signals," IEEE Trans. Aerosp. Electron. Syst., vol.25, no.6, pp.890-897, 1989.

[2] K. Lin and C.Q. Xu, "On binary sequence pairs with two-level periodic autocorrelation function," IEICE Trans. Fundamentals, vol.E93-A, no.11, pp.2278-2285, Nov. 2010.

[3] T. Jiang, L.T. Hou, and X.Q. Zhao, "The theory of perfect punctured binary array pairs," Acta Electronica Sinica, vol.32, no.2, pp.282286, Feb. 2004.

[4] C.L. Zhao, X.P. Peng, and C.Q. Xu, "Study on the characters of perfect ternary array pairs," Systems Engineering and Electronics, vol.31, no.5, pp.1030-1034, May 2009.

[5] C.Q. Xu and X.P. Peng, "A study on the methods for constructing perfect quatemary array pairs," Acta Electronica Sinica, vol.38, no.1, pp.6-12, Jan. 2010.

[6] H.D. Lüke, H.D. Schotten, and H. Hadinejad-Mahram, "Binary and quadriphase sequences with optimal autocorrelation properties: A syrvey," IEEE Trans. Inf. Theory, vol.49, no.2, pp.3271-3282, Dec. 2003.

[7] X.H. Tang and Jürgen Lindner, "Almost quadriphase sequences with ideal autocorrelation property," IEEE Signal Process. Lett., vol.16, no.1, pp.38-40, Jan. 2009.

[8] S.Y. Jin and H.Y. Song, "Note on a pair of binary sequences with ideal two-level crosscorrelation," ISIT., pp.2603-2607, July 2009.

[9] C.Q. Xu, "Difference set pairs and approach for the study of perfect binary array pairs," Acta Electronica Sinica, vol.29, no.1, pp.87-89, Jan. 2001.

[10] X.P. Peng and C.Q. Xu, "The constructions of several classes of binary sequence pairs with three-level autocorrelation," manuscript, submitted to Designs, Codes, and Cryptography, March 2011. 